\title{
La Plataforma Schoology en el Aprendizaje de la Matemática en Estudiantes Secundarios
}

\section{The Schoology Platform in Learning Mathematics in High School Students}

\author{
Eliana Rosa Avalos Monterrey \\ https://orcid.org/0000- 0002-5090-9453 \\ Universidad Cesar Vallejo, Perú \\ Shirley Mirella Vásquez León \\ https://orcid.org/0000-0003-1241-8191 \\ Universidad UNIFE, Perú \\ Flor de María Pereyra Romero \\ https://orcid.org/0000-0002-3536-2365 \\ Universidad Cayetano Heredia, Perú
}

Autor para correspondencia: rosaelianaavalos@gmail.com; shirvas1 @ hotmail.com; florcita_pereyra@hotmail.com

Fecha de recepción: 26 de febrero del 2020 - Fecha de aceptación: 09 de julio del 2020

\section{Resumen}

El estudio de naturaleza aplicada, tuvo como objetivo demostrar en qué medida, aplicar la plataforma Schoology influyó en los estudiantes del ciclo VI de secundaria en su aprendizaje correspondiente al área de matemática, haciendo uso de estrategias dinámicas y creativas, que les permitan desarrollar competencias matemáticas, como respuesta a la problemática estudiantil. La metodología utilizada responde a un enfoque cuantitativo, con un método hipotético deductivo, asimismo, se utilizó un diseño cuasi experimental, conformado por dos grupos llamados; pre test y post test; para lo cual se tomó una muestra no probabilística e intencional que comprendió a estudiantes de secundaria en un numero de 60, aplicándoles un cuestionario, de acuerdo a la prueba de normalidad. Se determinó la utilización de una prueba para muestras independientes, conocida como la T de Student para contrastar las hipótesis, antes y después de la utilización de estrategias dinámicas y creativas en la aplicación de la plataforma; llegando a la siguiente conclusión: El aplicar la plataforma Schoology influyó en forma significativa para el aprendizaje en las matemática en los estudiantes del VI ciclo de Secundaria, San Miguel, 2019, (T=4,737: gl= 58; p $<0,05)$.

Palabras claves: plataforma; programa; aprendizaje; matemática; competencia

\footnotetext{
Abstract

The study of applied nature, had as objective demonstrate to what extent applying the Schoology platform influenced students in high school VI cycle in their learning corresponding to 
mathematics subject, using dynamic and creative strategies, that allow them to develop mathematical competencies, as a response to student problems. The methodology used responds to a quantitative approach, with a hypothetical deductive method, likewise a quasi-experimental design was used to made up of two groups called; pretest and posttest; for which a non-probabilistic and intentional sample was taken that included high school students in a number of 60, applying a questionnaire to them, according to the normality test. The use of an independent samples, known as the Students T was determined to contrast the hypothesis; As a conclusion we can say: Applying the Schoology platform significantly influenced for the mathematics learning in the high school students of the VI cycle in San Miguel, 2019. ( $\mathrm{T}=4,737: \mathrm{gl}=58 ; \mathrm{p}<0,05)$.

Key words: platform; program; learning; mathematics; competence

\section{Introducción}

La necesidad de enfrentar retos del sistema educativo peruano, ha implicado que propongan la utilización de estrategias en las instituciones educativas, acorde al avance científico y tecnológico, dejando de lado las estrategias expositivas o meramente memorísticas, que no promueve el análisis, deja de lado el pensamiento creativo; así como la falta de motivación para aprender, sabemos que en el proceso que se da en la matemática para la enseñanza-aprendizaje, la motivación e interés del docente por formar está directamente relacionado con el del estudiante que busca aprender; y si se da esta debe ser recíproca, se logrará un verdadero aprendizaje significativo; se requiere el diseño de una estrategia que logre la complementación de las clases de matemática en el aula y que permita a los docentes, la posibilidad de acompañar de manera permanente a los estudiantes, que se fomente su autonomía, fortalezca el aprendizaje y la utilización de recursos educativos digitales, dentro y fuera del aula.

Siendo la matemática un área de desarrollo importante para el desarrollo integral del estudiante y enmarcado en el actual diseño curricular del (Minedu, 2019), donde se propone contenidos, estrategias y la utilización de recursos, para el análisis, la comprensión matemática; así como poder resolver problemas matemáticos, entre otros; representa para los estudiantes, dificultades, miedos, que los induce a una disposición negativa para el aprendizaje, por lo cual se requiere de estrategias dinámicas y motivadoras, la matemática no solo va a aportar en lo intelectual, también lo hará en lo afectivo, esto se puede transmitir porque al tener objetivos, los cuales no son fáciles de conseguir, que el afecto por la veracidad, precisión y certeza, y a ello le sumamos la seguridad en nuestras capacidades podremos tener una matemática bien ordenada la cual está considerada muy importante en nuestra vida cotidiana. No buscamos que nuestros estudiantes sean matemáticos, lo que se quiere lograr es la mejora de sus capacidades matemáticas, como por ejemplo la resolución de problemas en su quehacer diario y en su mundo laboral, participando activamente en la dinámica de nuestra sociedad y puedan producir en su comunidad para alcanzar el entendimiento de lo que sucede no solo en nuestro entorno, también en el mundo.

La Unidad de Gestión Educativa -UGEL 03 (2019), emitió informes de la prueba ECE del año 2018, (Minedu, 2018) y de evaluación diagnóstica del 2019, señalaron los siguientes resultados en los diferentes niveles, el $54 \%$ su nivel es previo en un inicio, luego $24 \%$ se le ubica en el inicio, en proceso encontramos el $12 \%$ y finalmente logrado es un 10\%; donde a raíz de esos resultados, se propuso el análisis de los factores que determinaron estos resultados y la 
implementación de estrategias como alternativa a la problemática descrita; donde se observó que los alumnos tienen algún tipo de dificultad para lograr aprender la matemática debido a que no desarrollan sus habilidades para interpretación, comprensión matemática, al plantear y resolver problemas matemáticos propios de su vida cotidiana.

De la misma manera, el estudio tiene como base los lineamientos del (Minedu, 2019), que en el currículo nacional propone la utilización de la tecnología, como son las TIC, buscando que las áreas se desarrollen, habiéndose considerado como un enfoque transversal, y de esta manera optimizar que los estudiantes logren un aprendizaje dentro de las instituciones educativas locales y distritales en el área de matemática; especialmente en aquellas donde se obtuvieron resultados en su aprendizaje, en niveles por debajo de lo esperado en el área de matemática, por lo que las TIC buscan integrar, engrandecer y evolucionar la educación, pero es necesario que los estudiantes tengan acceso a la tecnología, porque pueden existir muchos programas y plataformas que ayuden al aprendizaje de los estudiantes y que todos tengamos la llegada universal, con lo que buscaremos disminuir la desigualdad tanto en el aprendizaje como en la educación, aquí incluimos a los facilitadores los cuales deben tener las capacidades formativas para poder elaborar actividades para engrandecer las capacidades y fomentar el aprendizaje de sus estudiantes.

El estudio se desarrolló en la I.E. Rosa Irene Infante de Canales del distrito de San Miguel, que cuenta con una infraestructura y ambiente adecuados, con dos salas de cómputo, bien implementadas; donde se observa que los estudiantes están habituados a resolver prueba de manera mecánica, se limitan a marcar respuesta y no desarrollan o resuelven problemas, siendo un fiel reflejo de las estrategias utilizadas por el docente de matemática, con estrategias memorísticas y tradicionales; observándose también falta de atención y motivación en los estudiantes; quienes creen a la matemática como una asignatura muy cansada y tediosa; ante dicha problemática, se procura utilizar la plataforma Schoology para aprender la matemática óptimamente, de una manera progresiva ascendente y detectando la necesidad de cada estudiante como asimila su aprendizaje.

Después de haber descrito la realidad problemática, se formularon problemas, entre los que se tiene el problema general: ¿De qué forma aplicar la plataforma Schoology incide en el aprendizaje de la matemática?; asimismo, problemas específicos, como: 1) ¿De qué forma aplicar la plataforma Schoology influirá en el aprendizaje al resolver problemas cuantitativos en los estudiantes del VI Ciclo? 2) ¿De qué forma aplicar la plataforma Schoology influirá en el aprendizaje al resolver problemas de regularidad en los estudiantes del VI Ciclo? 3) ¿De qué forma aplicar la plataforma Schoology influirá en el aprendizaje al resolver problemas de localización en los estudiantes del VI Ciclo? 4) ¿De qué forma aplicar la plataforma Schoology influirá en el aprendizaje al resolver problemas de gestión en los estudiantes del VI Ciclo?

También se determinaron los objetivos, cuyo objetivo general fue: Establecer en qué medida utilizar Schoology influye en los estudiantes del VI de secundaria San Miguel 2019 para el aprendizaje de la matemática; y luego se determinó nuestros objetivos particulares y específicos: 1) Demostrar en qué medida la plataforma Schoology influye para resolver problemas cuantitativos en los estudiantes de VI ciclo. 2) Demostrar en qué medida la plataforma Schoology influye en la resolución de problemas de regularidad en los estudiantes de VI ciclo. 3) 
Demostrar en qué medida la plataforma Schoology influye para resolver problemas de localización en los estudiantes de VI ciclo. 4) Demostrar en qué medida la plataforma Schoology influye para resolver problemas de gestión en estudiantes de VI ciclo.

El estudio es importante porque, permite demostrar el efecto de aplicar la plataforma Schoology que influye en los estudiantes del nivel secundario (VI ciclo) en su aprendizaje en el área de matemática, donde dicha plataforma cumple con los requisitos de un programa educativo para el favorecimiento y logro de competencia en el área matemática; también es importante porque servirá a la comunidad educativa como un recursos didáctico, permite la utilización de las herramientas que ofrece, logrando simular un aula presencial, aplicando estrategias innovadoras y motivadoras para el aprendizaje de la matemática a través de la plataforma Schoology; de la misma manera se utilizan instrumentos que permitirán lograr la eficacia y eficiencia de la aplicación en la plataforma informática, contribuyendo a la mejora en el campo educativo; donde los resultados evidenciará la efectividad de la aplicación de dicha plataforma.

\section{Estudios acerca de plataformas virtuales y su aplicación en el área matemática}

De los estudios realizados acerca de la plataformas virtuales, (Angulo J., 2019), afirmo que este tipo de plataformas permiten a los estudiantes poder realizar un trabajo cooperativo de manera idónea y con ello la optimización del aprendizaje, además refirió las ventajas de la aplicación de la plataforma para el diseño gráfico, su investigación se hizo con la finalidad de determinar el impacto de uso de estas plataformas educativas pero a nivel superior, sin embargo fue un estudio transversal, no experimental y con un enfoque cuantitativo, realizado mediante una encuesta a los facilitadores que laboran con alguna plataforma educativa y cómo impacta en su práctica diaria pedagógica, siendo el resultado positivo; de la misma manera, (Sánchez \& Zegarra, 2017), señalaron la utilidad de las plataformas virtuales como el móvil y otros recursos informáticos para fomentar la investigación en los estudiantes, señalando la importancia de una permanente transmisión de conocimientos a través de una educación virtual, en la actualidad es indiscutible el rol destacado de las tecnologías y que se relaciona en los diferentes aspectos en nuestra vida en general, y muy particularmente en la educación, es necesario reconocer que la administración del intelecto se da gracias a la accesibilidad de muchos recursos tecnológicos, las cuales van a eliminar las restricciones o barreras existentes, convirtiéndose en instrumentos de administración de entendimiento.

De la misma manera, (Molina, Sebastiá, \& Sanchez, 2018), en un estudio sobre plataformas virtuales, señalaron que en la educación es importante el uso de la tecnología informática, se ha venido implementando de manera progresiva en las diferentes instituciones educativas, porque nos brinda muchas ventajas, tanto para el estudiante, así como para el profesor. Además, refiere que la plataforma Schoology es una herramienta que tiene la capacidad de dar la respuesta adecuada sobre lo que necesita el estudiante en nuestra actualidad, confiriéndole un papel decisivo e importante en el proceso de enseñar-aprender.

Definitivamente usar la tecnología, ha logrado una superioridad en su aprendizaje tanto en el alumno como en el docente, sin embargo esto se implementó en forma progresiva en las diferentes instituciones educativas; diferentes autores propusieron haber realizado experiencias con la utilización del Schoology, que permite al docente realizar un mejor acompañamiento para 
lograr que los estudiantes aprendan tanto en el área de matemáticas como en otras, lo que aporta esta plataforma es realizar un seguimiento al estudiante, para que su proceso de aprendizaje lo haga en forma independiente y tenga que realizar una autoevaluación del aprendizaje logrado, favoreciéndolo, sin embargo encontraremos que muchos de ellos no tendrán el interés por el cual puedan valorar este tipo de estudio.

Por otro lado, (Vargas, 2017), en su estudio, demostró que el uso de plataformas virtuales logra fortalecer hábitos de estudio, debido a que permite que los estudiantes organicen horas de estudio por áreas, así como tener el apoyo necesario que favorezca su aprendizaje, lo que busco la investigación es darle una valoración a los estudiantes por el uso de plataformas educativas digitales a personas que en su mayoría son trabajadoras y no pueden recibir clases presenciales, por lo cual el uso de la tecnología y plataformas es de suma importancia para lograr el aprendizaje deseado; que concuerda con la investigación de (Simanca, Abuchar, \& Velazco, 2017), quienes lograron elaborar un software que les permitió identificar y reconocer casos y solución de factorización de trinomios, concluyendo que las plataformas virtuales son de mucha utilidad en la cual los alumnos alcancen el aprendizaje en el área de matemática.

También, (Tuñoque, 2019), diseñó un programa para la utilización de estrategias didácticas con el Geogebra para resolver problemas, donde concluyó de la necesidad que los docentes utilicen estrategias innovadoras como plataformas virtuales y aplicaciones, permitiendo que nuestros estudiantes adquieran habilidades y capacidades con la finalidad de poder lograr la resolución de problemas matemáticos propios de su vida cotidiana; ello coindice con los hallazgos de (Goñi, 2018), quien demostró que la utilización de la plataforma Chamilo, mejoró de forma significativa que los alumnos de primer grado obtengan en el área de las matemáticas su aprendizaje deseado; resaltando las bondades en dicha plataforma, que permite al estudiante transformar espacios de enseñanza, organizar los temas a tratar, fomentar el autoaprendizaje y el aprendizaje colaborativo, también señaló que la utilización de un recurso tecnológico dentro del proceso que busca enseñar-aprender en el área de la matemática, se utilizó y se utiliza en las diferentes áreas, teniendo como finalidad la articulación entre las áreas; asimismo, señaló, que se debe tener en cuenta el logro de la competencia matemática en el logro de aprendizaje significativos, donde los docentes utilicen estrategias como plataformas virtuales para la optimización dela enseñanza.

\section{Entornos virtuales}

Debemos comprender que no solo basta con incorporar herramientas de índole tecnológico de información y comunicación en medio de aprendizaje, que está diseñado adecuadamente que aseguraría el éxito de esta. Es necesario que se tengan acciones hacia el estudiante en su proceso de aprendizaje, adquiriendo libertad para utilizar los recursos educativos que el facilitador puso a su orden y pueda desarrollar muchas habilidades y estrategias que le ayuden a planificar y regular su actividad de aprendizaje, nos referimos al aprender-aprender que es lo que se considera nuestra futura educación, basada en la rapidez de conseguir nuevos conocimientos y los medios que se tienen para poder permitir el ingreso de bastante información. Con estas condiciones, la pedagogía no solo trasmitirá información y conocimiento, también lograra que se pueda desarrollar la capacidad de producir y saber utilizarlos. Por lo cual se debe 
considerar que, formando a los estudiantes en los entornos virtuales, permitirán y propiciaremos un gran cambio en el rol de este.

(Silva, 2017), señaló que, los entornos virtuales, que se basan en modelos pedagógicos, sin un conjunto de elementos que logra guiar la manera de poder llevar el proceso que busca enseñar-aprender, para el cumplimiento de nuestra tarea (misión), a donde queremos llegar (visón) y las decisiones y acciones que realiza la institución educativa (principios estratégicos); el entorno virtual, orienta la actividad pedagógica, plasmada en el diseño curricular, así como elaborar materiales o medios didácticos a utilizar, discernir con cada proceso, y sobretodo el rol orientador del docente en la utilización dela herramientas informáticas, por lo que todos los involucrados deben tener una formación sobre la educación virtual, que incluirá saber manejar los entornos virtuales, en forma didáctica y especializada para lograr el aprendizaje del estudiante, también se debe involucrar en una capacitación del manejo de los entornos virtuales al estudiante, porque de nada sirve tener el manejo adecuado de la tecnología por el facilitador y el estudiante no lo pueda aprovechar por desconocimiento al utilizar estos entornos

Para (Mestre, Pérez, \& Valdés, 2017), el ambiente virtual, es un cumulo en el cual encontramos facilidades tanto informáticas y telemáticas que favorecen el intercambio y la comunicación en la búsqueda de información, con la finalidad de que se puedan desarrollar en actividades en el proceso de aprendizaje; es decir, es un aporte sustancial en los procesos pedagógicos, que con una utilización adecuada se optimizará el aprendizaje dentro y fuera del aula, sin embargo el objetivo principal de estos entornos es el autoaprendizaje del alumno, buscando también que los facilitadores puedan adquirir estrategias didácticas que le ayuden a futuro a utilizarlos en su labor pedagógica, esta se debe dar en todas las asignaturas donde se puedan usar medios informáticos y establecer los contenidos que se necesitaran para el aprendizaje del estudiante. Otra definición de un ambiente en el cual aprendemos, es donde consideramos desarrollar una naturaleza propicia para el aprendizaje, en el cual contemplaremos situaciones necesarias para ser implementadas en el currículo, es necesario que exista una relación fundamental entre el facilitador y el estudiante, es importante la planificación y estructura en el aula, habrá patrones de comportamiento que serán desarrollados en ella, el papel que se dispone y las actividades que se realizaran.

(Gros \& Noguera, 2014), aplicó el término entorno virtual del aprendizaje, al desarrollo formativo virtual que se utilizó en las diferentes instituciones educativas; que también se relaciona con la gestión de los temas tratados para el aprendizaje (LCMS) entornos de gestión del aprendizaje, es decir, son entornos web que son usados como herramienta en la cual se planifica actividades que buscan formar, ya sea en line o virtual; es así que señaló, que este sistema se puede instalar en una intranet, que nos va a permitir la creación, aprobación, administración, almacenamiento, distribución y gestión de actividades de formación virtual.

\section{Utilización de la plataforma Schoology}

(Molina, Sebastiá, \& Sanchez, 2018), manifiesta en su investigación que el Schoology es presentado como un recurso alternativo para dar respuestas al estudiante actualmente, ya que puede tener un rol activo. Cuya finalidad era evaluar los efectos de una clase semipresencial con Schoology frente a la metodología tradicional, es de tipo cuasi experimental, demostrando sus 
resultados que el usar las herramientas es más efectivas que una clase tradicional, en los diferentes estudios da como resultado que Schoology está considerada entre las cinco primeras plataformas de educación en línea.

(Anchundia, Torres, \& Segovia, 2019), señaló que una plataforma educativa es un instrumento virtual, que permite el desarrollo de la capacidad de interacción con fines pedagógicos; asimismo, se le considera una contribución a la transformación de los procesos que buscan lograr el aprendizaje, siendo una alternativa importante, o una estratega innovadora complementara en la educación tradicional, hoy estas plataformas casi en su totalidad son programas de software o equipos de hardware que tienen que cumplir algunos requerimientos y características con el fin de lograr los objetivos.

Así, (Chávez, 2019), señaló que Schoology es una plataforma gratuita de aprendizaje virtual gratuita, que sirve de acompañamiento tecnológico y que se puede implementar en los salones de clase en forma sencilla y de fácil utilización para lograr el aprendizaje deseado; su funcionamiento es como una estructura (red) social que se da entre los facilitadores (docentes) y estudiantes, en las cuales se genera diferentes foros como los debates, comunidades de alumnos que se pueden dividir en grupos, espacios de evaluación y complementar con otros recursos informáticos como Google drive , Edmodo; la cual en los sistemas virtuales la consideran el Facebook educativo, debido a que su uso es simple y de fácil acceso y se pueden crear comunidades con los nombres de profesor, alumno y padre de familia, en esta interface se pueden colgar clases presenciales, pero comparándola con Schoology no es muy potente, Moodle; que es considerado principalmente una plataforma donde se encuentran cursos de libre distribución, conocido como un lugar educativo virtual, en la cual los facilitadores pueden crear grupos o comunidades para el aprendizaje, llamadas también LMS (Learning Management System), entre otros. Muchos estudiantes e incluso los facilitadores también coincidieron que el usar las plataformas no era lo ideal, porque era necesario la práctica, enfatizando que por medio de la tecnología (internet) o las plataformas no podían tener una vivencia total y consideraban que es un tiempo perdido y que lo usarían para dejar trabajos, sin embargo, consideraron que la plataforma Schoology era la que más les fascino.

Ante ello, (Méndez \& Gutiérrez, 2016), manifestó que, es muy importante visualizar que en los procesos de aprendizaje se tienen que utilizar la tecnología que se conocen como Tics (tecnología de la información y comunicación), esta ha significado un gran efecto positivo en su organización; es decir, se considera a la plataforma educativa, como un instrumento que fortalece el logro del aprendizaje a través de los contenidos que logran ser procesados de mejor manera por los estudiantes, es importante reconocer que muchos profesionales están interesados en el desarrollo de los procesos que nos llevan a trasmitir lo aprendido y el conocimiento, para lo cual dieron un gran avance en la creación de estrategias que sirven tanto para enseñar como para aprender. Según transcurre el tiempo, esto no disminuye, por el contrario, aumenta y como consecuencia evoluciona todos los modelos educativos, actualizando los programas curriculares según las necesidades del momento, dando un gran avance desenfrenado del conocimiento, no solamente a los facilitadores, esta es llevado hacia los estudiantes.

De la misma manera, (Anchundia, Torres, \& Segovia, 2019), señaló que la plataforma Schoology, permite a los docentes y estudiantes a adoptar por nuevas estrategias para lograr 
buenos aprendizajes; porque siendo una herramienta online, favorece el compartir recursos, desarrollando acciones para facilitar que los estudiantes logren su capacidad cognitiva se desarrolle adecuadamente. Schoology es una plataforma virtual dinámica y cuenta con herramientas, que permiten realizar acciones de manera sencilla del proceso de enseñar-aprender; permite cuatro roles: estudiantes, docente, padres de familia y administrador, contando con herramientas que logran complementar su utilidad en el aula de clases, gracias a esta plataforma de fácil uso va a permitir a los facilitadores a la creación de un sin número de tareas con las cuales busca mejorar la motivación el curso y realizar un seguimiento del progreso de lo aprendido por el estudiante, esta plataforma le permitirá que el facilitador pueda agregar usuarios según corresponda a las asignaturas correspondientes, también se puede crear perfiles, organizara grupos de discusión y debate; nos permite agendar un calendario en simultaneo con todos los alumnos; poder corregir, subsanar y escribir recomendaciones sobre la tarea de los alumnos, sin la necesidad de realizarlo en forma repetitiva y por ultimo poder calificar de forma sencilla e individual con diferentes escalas personalizadas.

(Area, Hernández, \& Sosa, 2016), resalta para utilizar de forma adecuada las TIC lo siguiente; 1 . En la cual propone que lo notorio siempre sea lo educativo y no lo tecnológico, de aquí importara que el facilitador al planificar la utilización de las TIC, priorizara lo que el estudiante deba aprender y como la tecnología lo ayudara a mejorar su aprendizaje en el aula; 2. Como el facilitador debe conocer que las TIC no darán un efecto asombroso en el aprender del estudiante, lo que se debe priorizar es la motivación y estimulación que se logre transmitir al estudiante, para que mejore el rendimiento o crezca la atención por su aprendizaje; 3 . El facilitador utilizará estrategias que buscaran promover la recepción del aprendizaje por parte del estudiante, descubriendo nuevos conocimientos; 4. Se buscara utilizar las TIC en donde el estudiando debe aprender haciendo cosas con ayuda de la tecnología, esto lo hará incentivando a la búsqueda de datos, la manipulación de objetos digitales y la creación de información en formas distintas.

Entre los modelos teóricos acerca de la utilización de plataformas virtuales, se tiene Modelo del uso de las tic y el rol docente. (Hernandez, 2017), señaló que la falta interés en el manejo de las tecnologías o estrategias adecuadas en las aulas, es debido a que el facilitador se siente amenazado de perder su rol protagónico en el proceso en el cual se busca enseñaraprender, y/o que nuestros alumnos logren superarlo en el manejo de recursos tecnológicos. Asimismo, (Calle, 2014), señaló que la función del facilitador en el uso y manejo de la tecnología, cambia significativamente, por cuando se convierte en orientador de la búsqueda de la información apoyándose en las tic, dejando de ser el que poseía toda la información y conocimientos; ello implica la necesidad que los docentes deben actualizarse sobre el uso de herramientas informáticas y se logren los objetivos propuestos.

Teoría conductista-cognitivista-constructivista- (Cabero \& Llorente, 2015), señaló que se tiene la percepción que las tic son estímulos que permite desarrollar una respuesta específica a través del refuerzo, donde se destaca como tecnología a la enseñanza programada, en otras palabras debemos decir que la teoría conductista es el conocimiento memorístico, que muchas veces el facilitador utilizo apoyo y sancionó, para lograr que el estudiante consiga tener procedimientos y definiciones que busquen eliminar lo que no se desea; de la misma manera, señaló que las tics, son vistas como recursos válidos que favorecen el aprendizaje, ya que 
motivan a los estudiantes a participar, generando programa y sistemas donde desarrollan su capacidad cognitiva; asimismo, la teoría constructivista, logra potenciar que el estudiante se comprometa activamente, motivando su participación, interacción, retroalimentación y lo conecta con su entorno real.

Teoría conectivista. (Duke, Harper, \& Johnston, 2013), estableció que los estudiantes intentan el alcance de la comprensión mediante tareas que necesita un significado, donde se acepta la existencia del significado, y el reto del estudiante es, reconocer los patrones que se encuentran ocultos, es decir, la decisión sobre el significado y la formación de conexiones, son de manera principal la actividad central del aprendizaje. Sin embargo, de acuerdo a (Cabero \& Llorente, 2015), se le critica al conectivismo, considerada una guía educativa o un camino pedagógico a seguir, el cual se adapta o complementa a la realidad de la web 2.0 y la estructura que se da en internet conocida como redes sociales. A nuestro pensar el conectivismo se basa en como nosotros aprendemos por intermedio de nuestro comportamiento, por lo cual comenzamos a construir aprendizajes en nuestro día a día y alcanzar significativamente un aprendizaje, todos estos componentes tienen que estar vinculados con los últimos avances en las TIC, no podemos dejar de estar conectados a la tecnología ya que su avance es exponencial, y desconocer esta, nos volvería unos iletrados de la tecnología, es por eso que en forma diaria tenemos algo nuevo y distinto por aprender que nos facilita la vida, aquí encontramos a las aplicaciones en los celulares, el saber manejar una computadora y las tecnologías como un proyector entre otros.

Teoría del aprendizaje oblicuo. (Cobo \& Moravec, 2011), señalaron que consiste en el aprendizaje a través de la tecnología en cualquier momento y en cualquier lugar; es decir, que la enseñanza no solo sucede en el salón, sino también en cada uno de los hogares, cuando se juega, en el centro de labores, en la biblioteca, entre otros, implica la existencia de un escenario de aprendizaje invisible. Ante ello, es importante, resaltar que la conceptualización del aprendizaje es diferente a las de los siglos anteriores, dejando de lado el aprendizaje memorístico a un aprendizaje de conexión, mezcla y estructuración de la información.

A la vez, se mencionan las características de la plataforma Schoology, que de acuerdo a lo manifestado por, (Aldheleai, Ubaidullah, \& Alammari, 2017), éstas se encuentran en la nube, es de tipo gratuito, y ofrece un servicio de mensajería/email, permite la grabación de audios, se logra individualizar el dominio de cada curso y grupo, ofrece datos estadísticos, reportes de evaluación; es de fácil acceso para los cursos, con seguridad en los datos, se actualiza de manera permanente la tecnología utilizada, permite la participación de estudiantes y docentes con facilidad. (Jimenez, y otros, 2018), señalaron que el sistema de gestión LMS del Schoology, es una aplicación software que se basa en la web, utilizándose para la planificación, diseño y evaluación de procesos de aprendizaje específico, permitiendo al docente crear, enviar contenido y la evaluación del estudiante. 


\section{Figura 1.}

$L M S$

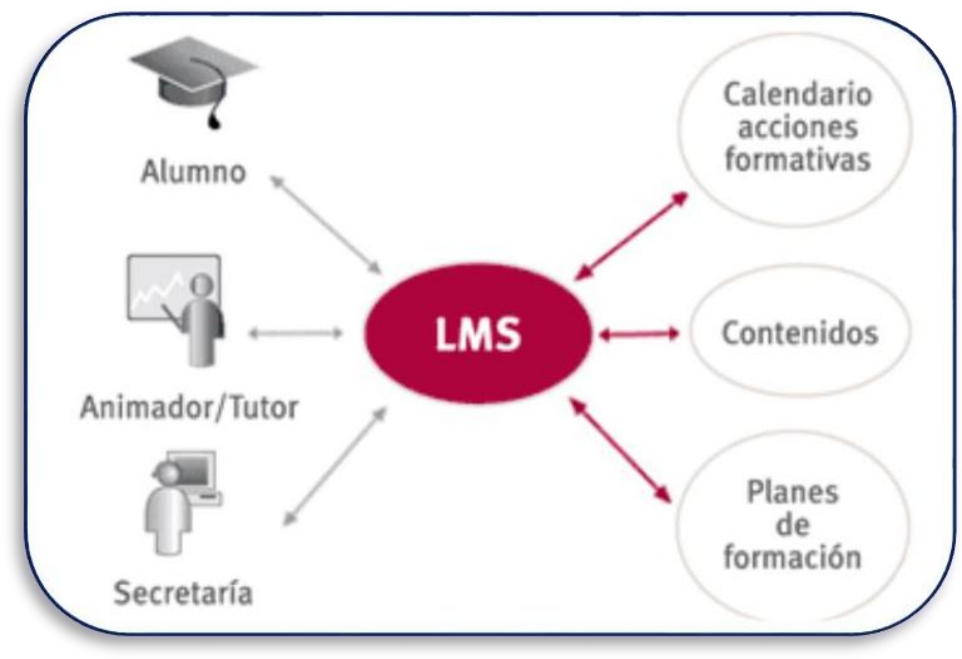

\section{Aprendizaje de la matemática en educación secundaria}

A través de la historia conocemos que cuando hablamos de matemática, el estudiante se asusta y considera que es una asignatura con la cual luchara, muchas veces hemos escuchado a los jóvenes estudiantes decir que nunca podre aprender y resolver diferentes problemas del área de matemática, podemos escuchar en las asignaturas de algebra, trigonometría entre otras, el escuchar esto es común en los salones de clase y los estudiantes dirán que para que me servirá, para lo cual también escucharemos en forma continua que lo necesitaras en el momento menos esperado, tal vez para un trabajo o te dirán que mejorara que tengamos un pensamiento crítico del cerebro, estas respuestas pueden ser consideradas buenas y son bien intencionadas, pero que en la realidad, la práctica de la matemática siempre la vamos a tener en nuestra vida diaria, en los diferentes aspectos.

Al respecto del aprendizaje de la matemática, (Bothia, 2017), citó a Ausubel, Bruner, Gagné y Vigotsky, quienes también trataron, como se aprende la matemática y observaron la actitud de los diferentes estudiantes cuando realizaron alguna actividad matemática, dejando de lado la conducta observable, considerando condicionamientos externos. Es decir, resaltando los mecanismos cognitivos del estudiante y de cómo se enfrenta al cometer algunos errores cuando realiza una determinada tarea. También observaron que dentro del aprendizaje van a encontrar muchas dificultades para solucionar los problemas, sin embargo, utilizan principios adquiridos en forma anterior, combinando ya lo aprendido para buscar la solución de un problema. Asimismo, (Zapata, 2015), señaló que el aprendizaje es el desarrollo cambiante en una persona, en forma permanente debido a que día a día tiene nuevas vivencias y logra una nueva experiencia, por lo que se considera que la capacidad cognitiva del estudiante evoluciona conforme pasa el tiempo, por lo cual a mayores vivencias van a traer consigo nuevos conocimientos; de la misma manera, para (Sánchez A. , 2015), refiere que es el proceso a través del cual se logra adquirir habilidades, logrando asimilar la información recibida, o adoptando una nueva o mejor estrategia del entendimiento y la acción; refiere también que en la actualidad, el mundo en que vivimos es muy cambiante y estos van a pasos acelerados, encontramos la 
intensificación de los conocimientos, que la ayuda de la tecnología nos abre la puerta en forma rauda a muchos cambios y por ende a nuevos conocimientos; ante esto encontramos que la educación es formadora de estudiantes que tienen grandes retos de investigación, adaptándose a las exigencias de nuestra sociedad actual. Conocemos que la matemática el día de hoy es considerada el resultado de un desarrollo largo y que en muchas ocasiones ha tenido confluencia con la filosofía, con el propósito de explicarla el universo, acorde a una interpretación racional que la matemática nos aporta. En tal sentido se tiene un progreso en la materia expuesta en un inicio por la lógica aristotélica, que plantea la unión del modelo matemático, más a la obligación de poder expresar el mundo que a una narración de índole, específicamente matemática. En consecuencia, la matemática nace de la necesidad de dar las explicaciones necesarias con respecto al entorno que se da en las diferentes relaciones que existen en la naturaleza, ello se debe a la capacidad de esta ciencia al interactuar con los diferentes elementos que componen nuestra naturaleza y el intelecto del ser humano, de aquí parte su íntima relación con la filosofía que en un principio es considerada más técnica que ciencia, para establecer que exista una conformidad entre el entorno y el entendimiento humano.

Así también, (Llufire, 2018), definió que es la capacidad para la operación con un lenguaje matemático que permita el fortalecimiento de las capacidades del pensamiento ordenado, razonamiento, argumentación, comunicación en la cual se utilizan otros códigos, modelamiento con diferentes situaciones de problemas, interpretación del lenguaje ya sea formal y simbólico, así como la resolución de problemas; señalando que es aquel donde los estudiantes realizan, luego de que los docentes inician las actividades partiendo de considerar el conocimiento previo que se relaciona con los contenidos de las matemáticas que toma como ejemplo presentando un problema que no podría resolverse mediante dichos saberes y provoca a los estudiantes, el interés de buscar nuevas formas para poder resolver soluciones inmediatas a su problema.

También, de acuerdo al currículo nacional, la enseñanza dentro de la matemática, está enmarcada a través de competencias, que son procesos complejos de esfuerzo con motivación en las actividades, integra conocimientos del quehacer y el convivir, y realizar las tareas como un reto, empeño, creatividad y desenvolvimiento en un proceso metacognitivo. Para el (Minedu, 2016), es el dominio que posee el estudiantes para desarrollar sus capacidades en la resolución de un problema; supone concebir, espacios para el enfrentamiento y la valoración de posibilidades para su resolución; significando ello, la fijación del conocimiento, habilidades con que cuenta. De la misma manera, la (OCDE, 2016), señaló que es la disposición que permite dar respuesta a situaciones que se presenten, realizando de manera práctica, combinaciones de competencias y se logren acciones con eficacia.

Para el (FONDEP, 2018), refiere que son potencialidades propias de la persona, que ésta desarrolla a lo largo de su vida; macro habilidad general, talento o condición especial de la persona, especialmente de naturaleza mental, que permite una mejor actuación en su vida diaria. En síntesis, la capacidad matemática, es la capacidad cognitiva de los procesos mentales que implica ser parte del quehacer educativo; es decir, cuando se tenga alguna relación con la matemática, necesariamente se desarrolla en nosotros el razonar, el demostrar, la comunicación matemática y poder resolver problemas. 
Una competencia es cuando ejecutamos un conocimiento, funcionara como una habilidad que se enfrentara a algunas situaciones, en cualquier ámbito de la vida. Por tanto, lo que nos deja actuar frente a diversas posturas en la vida diaria con efectividad seria lo aprendido que está basado en sus competencias. Además lo primordial en un modelo educativo de este tipo es que el estudiante pueda aprender por competencias más que conocimientos, porque este proceso incluye un crecimiento constante de lo aprendido y empieza a demostrarlo en muchas áreas distintas, en otras palabras este modelo está orientado principalmente a lograr que los estudiantes tengan un mayor rendimiento, es decir está abocado a los resultados, eliminando el paradigma en la educación tradicional basado en lo memorístico, tanto de datos como de información, que en repetidas ocasiones son innecesarias para la vida diaria. Aprendiendo competencias nos permitirá poder aplicarlas tanto en el entorno académico como en el laboral.

Asimismo, el (Minedu, 2016), determinó las dimensiones para el aprendizaje del área matemática; Dimensión 1: Logra resolver problemas de cantidad, donde el alumno logra solucionar enigmas y plantear problemas novedosos con el objetivo de lograr el entendimiento de nociones de número, sistema numérico, operaciones y propiedades; cuando realiza confrontaciones, logra esclarecer mediante analogías y ayuda de propiedades de casos particulares para desarrollar problemas, estableciendo el desarrollo de capacidades como: interpretación cuantitativa en expresiones numéricas, anuncia entendimiento de las diferentes operaciones utilizando estrategias y desarrollando procedimientos tanto de estimación y cálculo; dimensión 2: Soluciona la problemática que encuentra en su vida cotidiana y su entorno social; logrando que el estudiante pueda identificar y comparar equivalencias, generaliza la regularidad y el cambio de una magnitud a otra; asimismo, logra el desarrollo de las capacidades siguientes: interpretación de datos y características de expresiones algebraicas, logra manifestar su comprensión sobre expresiones algebraicas, identifica alguna estrategia y procedimiento para identificar reglas generales, logra deducir afirmaciones en las relaciones de cambio y equivalencia. Dimensión 3: Logra resolver problemas de forma, movimiento y localización; para que el estudiante pueda describir posición y movimiento de objetos de sí mismo en el espacio, que permite visualizar, interpretar y relacionar características de los objetos en dos y tres dimensiones, logra el desarrollo de capacidades, genera elementos que se apreciaran en formas geométricas y transformarlas, utilizando estrategias y planeando procedimientos en la orientación espacial, logra refutar afirmaciones sobre alguna relación geométrica; y dimensión 4: ayuda al estudiante poder resolver cierta complejidad de gestión de datos e incertidumbre: en el cual el estudiante pueda examinar temas de interés propio, basados en datos y pueda tomar decisiones, realizar pronósticos, desarrollar acciones, como: reproducir gráficos basados en datos, las cuales se pueden tomar estadísticamente o probabilísticamente, manejar alguna estrategia y procedimiento para la recopilación y procesamiento de datos, deducir conclusiones o decisiones teniendo como sustento la información recogida.

Se formularon hipótesis de investigación, y se tuvo como hipótesis general: La aplicación de la plata forma Schoology influirá en forma significativa en el aprendizaje de la matemática en los estudiantes del VI ciclo de la institución educativa Rosa Irene Infante de Canales de San Miguel; asimismo, las hipótesis específicas: 1) Aplicar la plataforma Schoology influirá en la competencia en la cual podrá resolver problemas de cantidad en forma significativa, en estudiantes del VI ciclo; 2) Aplicar la plataforma Schoology influirá en la competencia de resolver problemas de regularidad y cambio en forma significativa, en estudiantes de VI ciclo; 3 )

Esta obra se comparte bajo la licencia Creative Common Atribución-No Comercial 4.0 International (CC BY-NC 4.0) Revista de la Universidad Internacional del Ecuador. URL: https://www.uide.edu.ec/ 
Aplicar la plataforma Schoology influirá en la competencia para resolver problemas de forma, movimiento y localización en forma significativa, en los estudiantes de VI ciclo, 4) Aplicar la plataforma Schoology influirá en la competencia de resolver problemas sobre gestión de datos e incertidumbre de forma significativa, en los estudiantes del VI ciclo.

\section{Método}

Se utilizará un enfoque en el cual se podrán tomar datos con los cuales se podrán contrastar las hipótesis, a este se le conoce como cuantitativo, para lo cual tendremos como base un tipo de medición por números y se podrá establecer un análisis en forma estadística, por lo cual es necesario que se obtenga una muestra, que puede ser en forma aleatoria o discriminada, pero que es representativa dentro de nuestra población de estudio.

El diseño de investigación es cuasi experimental, la cual lo conforman dos grupos, pre test y post test, esto implica que la variable independiente pueda ser manipulada, para medir la variable dependiente y controlar las variables intervinientes; decir, el grupo experimental recibe el tratamiento, más no el grupo control, cuyo esquema es

$$
\begin{array}{cccc}
\text { G.E } & \mathrm{O}_{1} & \mathrm{X} & \mathrm{O}_{3} \\
\text { G.C } & \mathrm{O}_{2} & -- & \mathrm{O}_{4}
\end{array}
$$

La población estuvo conformada por 393 alumnos que comprenden 13 secciones de secundaria de la Institución Educativa Rosa Irene Infante de Canales, del distrito de San Miguel; y tuvo una muestra no probabilística e intencional, que comprende 60 alumnos de primer año de secundaria, dividido en dos grupos: 30 estudiantes del aula A, grupo control y 30 estudiantes del aula B, grupo experimental.

Utilizamos la técnica de investigación más utilizada, la encuesta y como instrumento una prueba tipo encuesta para medir la enseñanza en las matemáticas, la cual fue debidamente validada y para ello se aplicó un piloto como prueba a 30 alumnos y por ser un instrumento dicotómico se utilizó el estadístico KR20 y se obtuvo un coeficiente de confiabilidad 0,958.

Se realizó la recolección y procesamiento de datos, de la siguiente manera:

Se aplicó el test de matemáticas a dos secciones, de la siguiente manera: la sección 1, fue $1^{\circ} \mathrm{A}$, denominado grupo experimental y la sección 2 es $1^{\circ} \mathrm{B}$ determinado como grupo control.

En la semana 1, de acuerdo al cronograma del proyecto, se aplicó la pre prueba de matemáticas con alternativas de opción múltiple, el tiempo de duración estuvo estimado en 45 minutos a los grupos. Fue aplicado a 30 estudiantes de cada grado.

A partir de la semana 2, se trabajó en la plataforma Schoology con la sección 1, correspondiente al $1^{\circ} \mathrm{A}$, tratamiento para evidenciar los efectos en el aprendizaje de las matemáticas. 
Se elaboraron 10 sesiones de trabajo con dos horas semanales de $45 \mathrm{~min}$, en el área de matemática. Se organizó de la siguiente manera: Iniciamos en el mes de Setiembre y se desarrollaron 3 sesiones por mes haciendo uso de plataforma Schoology. Las estrategias empleadas han sido la asignación de tareas, elaboración de organizadores visuales, construcción de gráficos e interpretación, la participación de debates, evaluaciones en línea

La primera semana de diciembre se aplicó la prueba de salida a los dos grupos control y experimental que fue la misma que uso al inicio con la finalidad de establecer correlaciones pertinentes entre los grupos mencionados.

De la misma manera, se utilizó la prueba de normalidad de Shapiro-Wilk, cuyos resultados determinaron el uso de la prueba T de Student para la contratación de las hipótesis, y obteniendo los siguientes resultados:

Tabla 1.

Prueba de hipótesis - T de Student

\begin{tabular}{lllll}
\hline Hipótesis & T & Sig. (bilateral) & Gl & Decisión \\
\hline General & 4,737 &, 000 & 58 & Rechaza $\mathrm{H}_{0}$ \\
Específica 1 & 2,828 &, 006 & 58 & Rechaza $\mathrm{H}_{0}$ \\
Específica 2 & 2,955 &, 005 & 58 & Rechaza $\mathrm{H}_{0}$ \\
Específica 3 & 3,437 &, 001 & 58 & Rechaza $\mathrm{H}_{0}$ \\
Específica 4 & 3,320 &, 002 & 58 & Rechaza $\mathrm{H}_{0}$ \\
\hline
\end{tabular}

Fuente: Elaboración propia

En la tabla 1, los resultados obtenidos tienen valores $\mathrm{p}<0,05$ a un grado de libertad 58, por lo que se rechazan las hipótesis nulas en todos los casos; ello indica la aceptación de las hipótesis alternas relacionadas a la variable aprendizaje de la matemática y las competencias que se relacionan con sus respectivas dimensiones.

\section{Conclusiones}

La aplicación de la plataforma Schoology influye significativamente en el aprendizaje de la matemática en los estudiantes del VI ciclo de secundaria San Miguel 2019. (T=4,737: gl= $58 ; \mathrm{p}<0,05)$.

La aplicación de la plataforma Schoology influye significativamente en el aprendizaje de la matemática al resolver problemas de cantidad en los estudiantes del VI ciclo de secundaria San miguel, 2019. $(\mathrm{T}=2,828: \mathrm{gl}=58: \mathrm{p}<0,05)$.

La aplicación de la plataforma schoology influye significativamente en el aprendizaje de la matemática al resolver problemas de regularidad en los estudiantes del VI ciclo de secundaria San miguel, 2019. $(\mathrm{T}=2,955: \mathrm{gl}=58: \mathrm{p}<0,05)$.

La aplicación de la plataforma Schoology influye significativamente en el aprendizaje de la matemática al resolver problemas de localización en los estudiantes del VI ciclo de secundaria San miguel, 2019. $(\mathrm{T}=3,437: \mathrm{gl}=58: \mathrm{p}<0,05)$ 
La aplicación de la plataforma Schoology influye significativamente en el aprendizaje de la matemática al resolver problemas de gestión en los estudiantes del VI ciclo de secundaria San miguel, 2019. $(\mathrm{T}=3,320: \mathrm{gl}=58: \mathrm{p}<0,05)$.

\section{Bibliografía}

Aldheleai, H., Ubaidullah, M., \& Alammari, A. (Marxzo de 2017). ResearchGate - International Journal of Computer Applications. Recuperado el 27 de Diciembre de 2019, de Overview of Cloud-based Learning Management System. v. 162, n. 11, p. 41-46.: https://www.researchgate.net/publication/315174446_Overview_of_Cloudbased_Learning_Management_System

Anchundia, Y., Torres, R., \& Segovia, M. (Junio de 2019). ResearchGate. Recuperado el 29 de Diciembre de 2019, de La plataforma educativa Schoology: Impacto en el rendimiento académico de los estudiantes: https://www.researchgate.net/publication/333740663_La_plataforma_educativa_Schoolo gy_Impacto_en_el_rendimiento_academico_de_los_estudiantes

Angulo J., E. (26 de Julio de 2019). formacionib. Recuperado el 27 de Diciembre de 2019, de Trabajo colaborativo en Educación, fuerza en movimiento. República de Panamá IBERCIENCIA.: http://formacionib.org/noticias/?Una-fuerza-en-movimiento-el-trabajocolaborativo-en-educacion

Area, M., Hernández, V., \& Sosa, J. (Mayo de 2016). Revista científica iberoamericana de comunicación y educación. Recuperado el 23 de Diciembre de 2019, de Modelos de integración didáctica de las Tics en el aula. págs. 79-87: https://dialnet.unirioja.es/servlet/articulo?codigo $=5400275$

Bothia, L. (2017). Sistemas de Bibliotecas Unab. Recuperado el 26 de Diciembre de 2019, de Efecto del cuento en el proceso de aprestamiento de lectura y escritura en niños de 4 a 5 años [Recurso electrónico]. Universidad Autónoma de Bucaramanga, UNAB. Facultad de Educación: https://catalogo.unab.edu.co/cgi-bin/koha/opacdetail.pl?biblionumber $=167299 \&$ query_desc $=$ su $\% 3 A \% 22$ ESTRATEGIAS $\% 20$ EDUCAC IONALES\%22\%20and\%20su-to\%3AEstrategias\%20educacionales

Cabero, J., \& Llorente, M. (Abril de 2015). Red de Revistas Científicas de América Latina, el Caribe, España y Portugal. Recuperado el 29 de Diciembre de 2019, de Tecnologías de la Información y la Comunicación (TIC):escenarios formativos y teorías del aprendizaje. págs. 186-193.Corporación Universitaria Lasallist. Antioquia, Colombia: https://www.redalyc.org/pdf/695/69542291019.pdf

Calle, G. (06 de 2014). Repositorio Institucional Educativo- Universidad Autonoma del Caribe. Recuperado el 30 de Diciembre de 2019, de Las habilidades del pensamiento crítico durante la escritura digital en un ambiente de aprendizaje apoyado por herramientas de la web 2.0: http://repositorio.uac.edu.co/handle/11619/1442

Chávez, N. (18 de Diciembre de 2019). EDUNOVATIC 2019. Recuperado el 30 de Diciembre de 2019, de Percepción del uso de la plataforma de Schoology en estudiantes de educación superior. $\quad$ pag. 117 : http://www.edunovatic.org/wpcontent/uploads/2020/03/EDUNOVATIC19.pdf

Cobo, C., \& Moravec, J. (Diciembre de 2011). Red de Revistas Científicas de América Latina, el Caribe, España y Portugal. Recuperado el 15 de Diciembre de 2019, de Introducción al aprendizaje invisible: la (r)evolución fuera del aula. págs. 66-81. Universidad Autónoma 
Metropolitana Unidad Xochimilco.Distrito Federal, México: https://www.redalyc.org/pdf/340/34021066008.pdf

Duke, B., Harper, G., \& Johnston, M. (2013). Connectivism as a Learning Theory Critique: Persuasive Essay. Recuperado el 30 de Diciembre de 2019, de Connectivism as a Learning Theory for the Digital Age. Kaplan University, Ft. Lauderdale, FL: https://hetl.org/wpcontent/uploads/gravity_forms/2298b245759ca2b0fab82a867d719cbae/2013/01/Connectivism-hand-out.pdf

FONDEP. (2018). Minedu. Recuperado el 27 de Diciembre de 2019, de Sugerencias para la aplicación de las Orientaciones para el año escolar 2019 en el tema de la innovación educativa: http://www.fondep.gob.pe/wp-content/uploads/2019/03/Norma-Escolar-2019FONDEP.pdf

Goñi, F. (2018). Repositorio de la Universida Nacional Enrique Guzmán y Valle. Recuperado el 28 de Diciembre de 2019, de Plataforma Chamilo como herramienta e-learning y blearning en el aprendizaje de matemática en los estudiantes del ciclo avanzado del CEBA Rosa de Santa María. Universidad Nacional de Educación Enrique Guzmán y Valle. Lima, Perú.: http://repositorio.une.edu.pe/handle/UNE/1666

Gros, B., \& Noguera, I. (24 de Julio de 2014). RELATEC: Revista Latinoamericana de Tecnología Educativa. Recuperado el 27 de Diciembre de 2019, de Indicadores para la construcción de prácticas colaborativas en entornos virtuales de aprendizaje.: https://dialnet.unirioja.es/servlet/articulo?codigo $=4835389$

Hernandez, R. (06 de Marzo de 2017). Revista de Psicología Educativa. Recuperado el 28 de Diciembre de 2019, de Impacto de las TIC en la educación: Retos y Perspectivas. págs. 325 - 347: http://revistas.usil.edu.pe/index.php/pyr/article/view/149

Jimenez, J., Pueo, B., Carbonell, J., Saiz, S., Penichet, A., Molina, N., y otros. (2018). RUA. Repositorio Institucional de la Universidad de Alicante. Recuperado el 30 de Diciembre de 2019, de Sistema de gestión del aprendizaje (LMS) como herramienta para la mejora del aprendizaje en Educación Superior.Págs. 3027-3032. Universidad de Alicante. Departamento de Didáctica General y Didácticas Específicas: http://rua.ua.es/dspace/handle/10045/91113

Llufire, M. (2018). Repositorio de Tesis UCV. Recuperado el 27 de Diciembre de 2019, de Noción de números y aprendizaje matemático en los estudiantes de 5 años de la I.E.I 6152, Villa María del Triunfo, 2015: http://repositorio.ucv.edu.pe/bitstream/handle/UCV/16353/Llufire_QMM.pdf?sequence= $1 \&$ isAllowed $=\mathrm{y}$

Méndez, A., \& Gutiérrez, D. (Diciembre de 2016). Libros y materiales gratuitos para enseñar y aprender. Recuperado el 19 de Dciembre de 2019, de Estrategias de enseñanza y aprendizaje. Una mirada desde diferentes niveles educativos. Red Durango de Investigadores Educativos A. C. Editado en México.: https://leagratis.blogspot.com/2019/02/estrategias-de-ensenanza-y-aprendizaje.html

Mestre, U., Pérez, F., \& Valdés, P. (Enero de 2017). ResearchGate. Recuperado el 29 de Diciembre de 2019, de Entornos virtuales de enseñanza aprendizaje.: https://www.researchgate.net/publication/277029818_Entornos_virtuales_de_ensenanza_ aprendizaje

Minedu. (2016). MINEDU. Recuperado el 29 de Diciembre de 2019, de Programa Curricular Nacional: http://www.dreapurimac.gob.pe/inicio/images/ARCHIVOS2017/a-educainicial/programa-inicial-16-marzo-web.pdf 
Minedu. (2018). UMC. Recuperado el 30 de Diciembre de 2019, de Resultados de la Evaluación censal de Estudiantes 2018: http://umc.minedu.gob.pe/resultados-ece-2018/

Minedu. (2019). Minedu. Recuperado el 29 de Diciembre de 2019, de Curriculo Nacional de la educación Básica: http://www.minedu.gob.pe/curriculo/pdf/curriculo-nacional-2016-2.pdf

Molina, N., Sebastiá, S., \& Sanchez, L. (Octubre de 2018). ReasearchGate. Recuperado el 27 de Diciembre de 2019, de Blended learning vía Schoology como alternativa a la clase magistral: Estudio de caso.: https://rua.ua.es/dspace/bitstream/10045/88087/1/2018-Elcompromiso-academico-social-113.pdf

OCDE. (2016). MINEDU. Recuperado el 29 de Diciembre de 2019, de El Perú en PISA 2015: http://umc.minedu.gob.pe/wp-content/uploads/2017/04/Libro_PISA.pdf

Sánchez, \& Zegarra. (26 de Julio de 2017). pirhua. Recuperado el 27 de Diciembre de 2019, de Aplicación del Programa Virtual LMS-Schoology para mejorar el rendimiento académico del área de Investigación en estudiantes de Maestría de la Universidad Autónoma del Perú. En IV Congreso Iberoamericano de Estilos de Aprendizaje. Universidad de Piura. : https://pirhua.udep.edu.pe/handle/11042/3146

Sánchez, A. (2015). Repositorio de Tesis, Universidad Andina Nestor Cáceres Velasquez. Recuperado el 30 de Diciembre de 2019, de Estrategias de Enseñanza y Aprendizaje Empleada por Docentes de Matemáticas y su Incidencia en el Rendimiento Académico de Estudiantes del Tercer Grado de Educación Secundaria en la Ciudad de Juliaca Año 2014: http://repositorio.uancv.edu.pe/handle/UANCV/782

Silva, J. (31 de Marzo de 2017). RED. Revista de Educación a Distancia. Recuperado el 21 de Diciembre de 2019, de Un modelo pedagógico virtual centrado en las E-actividades. Universidad de Santiago de Chile: https://www.um.es/ead/red/53/silva.pdf

Simanca, F., Abuchar, A., \& Velazco, S. (Junio de 2017). Redes de Ingeniería. Recuperado el 27 de Diciembre de 2019, de Las tic y el aprendizaje de los trinomios. Revista Electrónica Redes de Ingeniería, 199-207: https://revistas.udistrital.edu.co/index.php/REDES/article/view/12492/13096

Tuñoque, E. (28 de Noviembre de 2019). Repositorio Institucional. Recuperado el 27 de Diciembre de 2019, de Diseño de un programa de estrategias didácticas con el uso del software matemático GEOGEBRA, basado en el enfoque de resolución de problemas, que fundamente la mejora del desarrollo de capacidades en el área de matemática en los estudiantes del $2^{\circ}$ g.E.S.: http://repositorio.unprg.edu.pe/handle/UNPRG/6599

Vargas, A. (31 de Octubre de 2017). Dialnet. Recuperado el 26 de Diciembre de 2019, de El uso de plataformas virtuales y su impacto en el proceso de aprendizaje en las asignaturas de las carreras de Criminología y Ciencias Policiales de la Universidad Estatal a Distancia de Costa Rica: https://dialnet.unirioja.es/servlet/articulo?codigo $=6228645$

Zapata, M. (2015). Red de Revistas Científicas de América Latina, el Caribe, España y Portugal. Recuperado el 30 de Diciembre de 2019, de Teorías y modelos sobre el aprendizaje en entornos conectados y ubicuos. Bases para un nuevo modelo teórico a partir de una visión crítica del "conectivismo"Education in the Knowledge Society, vol. 16, núm. 1, 2015, pp. 69-102.Universidad de Salamanca: https://www.redalyc.org/pdf/5355/535554757006.pdf 